\title{
The Finite Element Method With Nonuniform Mesh Sizes for Unbounded Domains*
}

\author{
By C. I. Goldstein
}

\begin{abstract}
The finite element method with nonuniform mesh sizes is employed to approximately solve elliptic boundary value problems in unbounded domains. Consider the following model problem:

$$
-\Delta u=f \quad \text { in } \Omega^{c}, \quad u=g \text { on } \partial \Omega, \quad \frac{\partial u}{\partial r}+\frac{1}{r} u=o\left(\frac{1}{r}\right) \text { as } r=|x| \rightarrow \infty,
$$

where $\Omega^{C}$ is the complement in $R^{3}$ (three-dimensional Euclidean space) of a bounded set $\Omega$ with smooth boundary $\partial \Omega, f$ and $g$ are smooth functions, and $f$ has bounded support. This problem is approximately solved by introducing an artificial boundary $\Gamma_{R}$ near infinity, e.g. a sphere of sufficiently large radius $R$. The intersection of this sphere with $\Omega^{C}$ is denoted by $\Omega_{R}^{C}$ and the given problem is replaced by$$
-\Delta u_{R}=f \quad \text { in } \Omega_{R}^{C}, \quad u_{R}=g \text { on } \partial \Omega, \quad \frac{\partial u_{R}}{\partial r}+\frac{1}{r} u_{R}=0 \text { on } \Gamma_{R} .
$$

This problem is then solved approximately by the finite element method, resulting in an approximate solution $u_{R}^{h}$ for each $h>0$. In order to obtain a reasonably small error for $u-u_{R}^{h}=\left(u-u_{R}\right)+\left(u_{R}-u_{R}^{h}\right)$, it is necessary to make $R$ large. This necessitates the solution of a large number of linear equations, so that this method is often not very good when a uniform mesh size $h$ is employed. It is shown that a nonuniform mesh may be introduced in such a way that optimal error estimates hold and the number of equations is bounded by $\mathrm{Ch}^{-3}$ with $C$ independent of $h$ and $R$.
\end{abstract}

1. Introduction. The finite element method has been studied extensively in connection with elliptic boundary value problems on bounded domains; see, e.g., [1]-[3] and the references cited there. In particular, it has been shown that optimal error estimates hold under suitable assumptions on the differential operator and the finite element subspace. It is the purpose of this paper to show that, by employing the finite element method with an appropriately graded mesh near infinity, analogous results hold for unbounded domains.

We consider the following model problem:

(1.1) $-\Delta u=f$ in $\Omega^{C}, \quad u=g$ on $\partial \Omega$ and $\frac{\partial u}{\partial r}+\frac{1}{r} u=o\left(\frac{1}{r}\right)$ as $r=|x| \rightarrow \infty$, where $\Omega^{C}$ is the complement in $R^{3}$ (three-dimensional Euclidean space) of a bounded domain $\Omega$ with smooth boundary $\partial \Omega, f$ and $g$ are smooth functions, and $f$ has bounded support. We shall approximate this problem by introducing a sphere

Received February 13, 1980; revised June 16, 1980.

1980 Mathematics Subject Classification. Primary 65N15, 65N30.

* The submitted manuscript has been authored under contract DE-AC02-76H00016 with the U. S. Department of Energy. Accordingly, the U. S. Government retains a nonexclusive, royalty-free license to publish or reproduce the published form of this contribution, or allow others to do so, for U. S. Government purposes. 
$\Gamma_{R}$ of sufficiently large radius $R$, as well as an approximate boundary condition on this sphere. We denote the region bounded by $\partial \Omega$ and $\Gamma_{R}$ by $\Omega_{R}$ and replace problem (1.1) by

$$
-\Delta u_{R}=f \quad \text { in } \Omega_{R}, \quad u_{R}=g \text { on } \partial \Omega \text { and } \frac{\partial u_{R}}{\partial r}+\frac{1}{r} u_{R}=0 \text { on } \Gamma_{R} .
$$

Problem (1.2) may be approximately solved using the finite element method, resulting in an approximate solution $u_{R}^{h}$ for each $h>0$. In order to obtain a suitable estimate for $u-u_{R}^{h}=\left(u-u_{R}\right)+\left(u_{R}-u_{R}^{h}\right)$, where $u$ and $u_{R}$ satisfy (1.1) and (1.2), respectively, it will be necessary to make $R$ large. In the usual finite element formulation with a uniform mesh size $h$, this results in an excessively large number of linear equations. We shall overcome this difficulty by grading the mesh systematically in such a way that the element mesh sizes become larger as the distance of the element from the origin increases. It will be proved that optimal error estimates for $u-u_{R}^{h}$ hold (in the sense to be described in Section 3 ), while the number of equations is bounded by $\mathrm{Ch}^{-3}$ with $C$ independent of $h$ and $R$. The results will follow by combining certain rates of decay estimates for the derivatives of $u$ with approximation theory.

We now outline the remainder of the paper. In Section 2, we introduce our notation and describe the boundary value problems under consideration, as well as a variational formulation of problem (1.2). The main result of Section 2 is an estimate for $u-u_{R}$ in both the energy norm and the $L^{2}$ norm. In Section 3, we describe the finite element method for solving problem (1.2) and obtain an estimate for $u_{R}-u_{R}^{h}$ in the energy norm when a uniform mesh size is employed. We shall see that this yields a suboptimal error estimate for $u-u_{R}^{h}$. In Section 4, we describe the mesh grading technique and obtain an optimal error estimate for $u-u_{R}^{h}$ in the energy norm. In Section 5, we obtain an optimal error estimate in the $L^{2}$ norm. The main results of the paper are embodied in Theorems 4.1 and 5.1.

For other references, treating problems in unbounded domains using the finite element method, see [2, pp. 276-280] and [4]-[6], as well as additional references cited there. The mesh grading technique has been employed in [7]-[10] to treat domains with corners.

2. The Boundary Value Problems. In this section, we shall investigate problems (1.1) and (1.2). We begin by defining some notation and formulating the given problem on an unbounded domain in $R^{3}$. We shall then formulate an approximate problem on a bounded domain and establish estimates for the difference between the solutions of these two problems.

We shall employ the usual notation for Sobolev spaces. In particular, suppose that $M$ is a nonnegative integer and $B$ is a subset of $R^{3}$. (We shall obtain our results in $R^{3}$ although the arguments carry over to $R^{2}$ in a straightforward manner.) Set

$$
|u|_{H^{M}(B)}=\left(\sum_{|\alpha|=M}\left\|D_{u}^{\alpha}\right\|_{L^{2}(B)}^{2}\right)^{1 / 2}
$$


and

$$
\|u\|_{H^{M}(B)}=\left(\sum_{s=0}^{M}|u|_{H^{2}(B)}^{2}\right)^{1 / 2},
$$

where $\alpha=\left(\alpha_{1}, \alpha_{2}, \alpha_{3}\right)$ with each integer $\alpha_{j} \geqslant 0,|\alpha|=\sum_{j=1}^{3} \alpha_{j}$, and $D^{\alpha}$ denotes the weak derivative of the real-valued function $u$. Thus, ||$_{H^{M}(B)}$ defines a seminorm, whereas \|\|$_{H^{M(B)}}$ defines a norm. Set $H^{M}(B)=\left\{u:\|u\|_{H^{M}(B)}<\infty\right\}$. An arbitrary point in $R^{3}$ will be denoted by $x=\left(x_{1}, x_{2}, x_{3}\right)$ in Cartesian coordinates. We shall also employ spherical polar coordinates, $(r, \omega, \theta)$, defined by $x_{1}=r \sin \theta \cos \omega$, $x_{2}=r \sin \theta \sin \omega$, and $x_{3}=r \cos \theta$.

We next define our boundary value problem as follows. Let $\Omega$ denote a bounded domain in $R^{3}$ with $C^{\infty}$ boundary $\partial \Omega$. Denote the complement of $\bar{\Omega}=\Omega \cup \partial \Omega$ by $\Omega^{C}$. We shall also require the sets $S_{R}$ and $\Omega_{R}$ given by $S_{R}=\{x:|x|<R\}$ and $\Omega_{R}=\Omega^{C} \cap S_{R}$. Suppose that $g \in C^{\infty}(\partial \Omega), f \in C^{\infty}\left(\overline{\Omega^{C}}\right)$, and $f$ has bounded support. (For the sake of simplicity, we are assuming more smoothness of $f, g$ and, $\partial \Omega$ than necessary.) Throughout the paper, we shall assume that $R$ is sufficiently large that $\Omega \subset S_{R}$ and $\operatorname{supp}(f) \subset \bar{\Omega}_{R}$. Our aim is to solve the boundary value problem given by (1.1). (The results and arguments of this paper go through without essential change if the Dirichlet boundary condition on $\partial \Omega$ is replaced by the Neumann condition.)

It suffices to consider the following boundary value problem:

$$
-\Delta u=f \quad \text { in } \Omega^{c}, \quad u=0 \text { on } \partial \Omega \text { and } \frac{\partial u}{\partial r}+\frac{1}{r} u=o\left(\frac{1}{r}\right) \quad \text { as } r \rightarrow \infty .
$$

To obtain (2.1) from (1.1), we observe that $g$ may be extended to a smooth function $G$ with bounded support. If $v$ satisfies (1.1), we set $u=v-G$ and observe that $u$ satisfies (2.1) with $f$ replaced by $f+\Delta G$. We now establish some important properties of the solution $u$ of (2.1) that will be useful throughout this paper.

LEMMA 2.1. Suppose that $u \in C^{\infty}\left(\Omega^{C}\right)$ satisfies (2.1). Then for $r=|x|$ sufficiently large and each multi-index $\alpha$, we have

$$
\begin{gathered}
D_{x}^{\alpha} u(x)=\sum_{n=1}^{\infty} \frac{a_{n}^{\alpha}(\omega, \theta)}{r^{n+|\alpha|}}, \\
\left|D_{x}^{\alpha} u(x)\right|^{2} \leqslant \frac{C}{r^{2(|\alpha|+1)}}\left(\oint_{\partial \Omega}\left|\frac{\partial u}{\partial n}\right|^{2} d s_{x}+\oint_{\Omega}|f|^{2} d x\right),
\end{gathered}
$$

and

$$
\left|\frac{\partial u(x)}{\partial r}+\frac{1}{r} u(x)\right|^{2} \leqslant \frac{C}{r^{6}}\left(\oint_{\partial \Omega}\left|\frac{\partial u}{\partial n}\right|^{2} d s_{x}+\int_{\Omega}|f|^{2} d x\right) .
$$

The constant $C$ is independent of $r$ and $u$.

Note that we shall often use the same letter $C$ to denote different constants when there is no danger of confusion.

Proof. (a) We may apply (2.1) and integration by parts to see that

$$
u(x)=\frac{1}{4 \pi}\left(\oint_{\partial \Omega} \frac{1}{\rho} \frac{\partial u\left(x^{\prime}\right)}{\partial n^{\prime}} d s_{x^{\prime}}-\int_{\Omega^{c}} \frac{1}{\rho} f\left(x^{\prime}\right) d x^{\prime}\right) \quad \forall x \in \Omega^{c},
$$


where $\rho=\left|x-x^{\prime}\right|, x^{\prime}=\left(r^{\prime}, \omega^{\prime}, \theta^{\prime}\right)$, and $n^{\prime}$ is the outward directed normal to $\partial \Omega$ at the point $x^{\prime}$. It may be seen (as, e.g., in [11]) that

$$
\frac{1}{\rho}=\frac{1}{r \sqrt{1-\frac{2 r^{\prime}}{r} \cos \psi+\left(\frac{r^{\prime}}{r}\right)^{2}}} .
$$

Here $\psi$ is the angle between the rays joining $x$ and $x^{\prime}$ to the origin, so that $\cos \psi=\cos \theta \cos \theta^{\prime}+\sin \theta \sin \theta^{\prime} \cos \left(\omega-\omega^{\prime}\right)$. Since $f$ has bounded support, we readily obtain (a) using (2.2) and (2.3).

(b) It is clear that we may differentiate under the integral sign in (2.2). Hence, (b) follows from (2.2), (2.3), and the Schwarz inequality.

(c) We again apply (2.2), (2.3), and the Schwarz inequality to see that (c) holds. Q.E.D.

We next establish the well-posedness of problem (2.1).

\section{LEMMA 2.2. There exists a unique solution $u \in C^{\infty}\left(\Omega^{C}\right)$ of problem (2.1).}

Proof. The existence of a solution $u$ of (2.1) is well known and may be established using the method of integral equations; see, e.g., [12] and the references cited there. The smoothness of $u$ follows from the smoothness of $f$ and $\partial \Omega$ using standard regularity theory for elliptic differential equations, [13]. The uniqueness of $u$ may be established as follows. Suppose that $f=0$ in (2.1) and apply integration by parts on $\Omega_{R}$ with $R$ large to obtain:

$$
O=\int_{\Omega_{R}}-u \Delta u d x=\int_{\Omega_{R}}|\nabla u|^{2} d x-\oint_{\partial S_{R}} u \frac{\partial u}{\partial r} d s_{x} .
$$

It follows from Lemma 2.1(a) that

$$
u(x)=O\left(\frac{1}{r}\right) \text { and } \quad \frac{\partial u(x)}{\partial r}=O\left(\frac{1}{r^{2}}\right) \text { as } r \rightarrow \infty .
$$

Hence, we may let $R \rightarrow \infty$ to see that $\int_{\Omega^{c}}|\nabla u|^{2} d x=0$. Thus, $u$ is a constant in $\Omega^{C}$ and $u=O(1 / r)$ as $r \rightarrow \infty$, so that $u=0$ in $\Omega^{C}$. We have thus proved uniqueness. Q.E.D.

Note. Even if $f \notin C^{\infty}\left(\Omega^{C}\right), u(x)$ is still infinitely differentiable outside of the support of $f$.

As we indicated earlier, we shall not approximate the solution of (2.1) directly but instead shall introduce an intermediate problem on a bounded domain. We thus consider the following problem:

$$
-\Delta_{R}=f \quad \text { in } \Omega_{R}, \quad u_{r}=0 \quad \text { on } \partial \Omega, \quad \frac{\partial u_{R}}{\partial r}+\frac{1}{r} u_{R}=0 \quad \text { on } \partial S_{R} .
$$

In view of the last equation in (2.4), it follows from Lemma 2.1(c) that

$$
\begin{gathered}
\max _{x \in \partial S_{R}}\left|\frac{\partial}{\partial r}\left(u(x)-u_{R}(x)\right)+\frac{1}{r}\left(u(x)-u_{R}(x)\right)\right|=\max _{x \in \partial S_{R}}\left|\frac{\partial u(x)}{\partial r}+\frac{1}{r} u(x)\right| \\
<\frac{C}{R^{3}}\left(\oint_{\partial \Omega}\left|\frac{\partial u}{\partial n}\right|^{2} d s_{x}+\int_{\Omega^{c}}|f|^{2} d x\right)^{1 / 2} .
\end{gathered}
$$

Our main goal in this section is to establish mean-square estimates for $u-u_{R}$ and $\nabla\left(u-u_{R}\right)$ over $\Omega_{R}$. First, however, we prove that problem (2.4) is well posed. 
LEMMA 2.3. There exists a unique solution $u_{R} \in C^{\infty}\left(\Omega_{R}\right)$ of (2.4).

Proof. First we prove uniqueness. Suppose that $u_{R}$ satisfies (2.4) with $f=0$ and note that

$$
\begin{aligned}
0 & =\int_{\Omega_{R}}-u_{R} \Delta u_{R} d x=\int_{\Omega_{R}}\left|\nabla u_{R}\right|^{2} d x-\oint_{\partial \Omega_{R}} u_{R} \frac{\partial u_{R}}{\partial r} d s_{x} \\
& =\int_{\Omega_{R}}\left|\nabla u_{R}\right|^{2} d x+\frac{1}{R} \oint_{\partial S_{R}}\left|u_{R}\right|^{2} d s_{x} .
\end{aligned}
$$

Hence, $u_{R}$ is a constant in $\Omega_{R}$ and $u_{R}=0$ on $\partial S_{R}$. Thus, $u_{R}=0$ in $\Omega_{R}$ and we have proved uniqueness. The remainder of the theorem now follows from [13, Theorem 2.1], in view of the smoothness of $f$ and $\partial \Omega$. Q.E.D.

In order to apply the finite element method to problem (2.4), we first express this problem in a variational formulation. Set

$$
H_{R}^{E}=\left\{u: u \in H^{1}\left(\Omega_{R}\right), u=0 \text { on } \partial \Omega\right\}
$$

and define

$$
\left\{\begin{array}{l}
a_{R}(u, v)=\int_{\Omega_{R}} \nabla u \cdot \nabla v d x+\frac{1}{R} \oint_{\partial S_{R}} u v d s_{x} \quad \forall u, v \in H_{R}^{E}, \\
\text { and } \\
\|u\|_{H_{R}^{E}}=\left(a_{R}(u, u)\right)^{1 / 2} \quad \forall u \in H_{R}^{E} .
\end{array}\right.
$$

LEMMA 2.4. (a) There exists a constant $C(R)$ such that

$$
\|v\|_{H^{1}\left(\Omega_{R}\right)} \leqslant C(R)\|v\|_{H_{R}^{E}} \quad \forall v \in H_{R}^{E}
$$

where $C(R)$ is independent of $v$ (but depends on $R$ in general),

(b) there exists a unique function $u^{1} \in H_{R}^{E}$ satisfying the equation

$$
a_{R}\left(u^{1}, v\right)=(f, v) \quad \forall v \in H_{R}^{E}
$$

and

(c) $u^{1}=u_{R}$, where $u_{R}$ is the solution of (2.4).

Proof. (a) This follows from Rellich's theorem as in [2, Theorem 1.2.1].

(b) This follows readily from (a); see, e.g., [2] or [3].

(c) This follows easily from (2.4), (2.6), and (b) using integration by parts. Q.E.D.

We are now ready to obtain estimates for $e_{R}(x)=u(x)-u_{R}(x)$, where $u$ and $u_{R}$ satisfy (2.1) and (2.4), respectively. For convenience, we set

$$
w_{R}(x)=\left(\frac{\partial}{\partial r}+\frac{1}{R}\right) u(x) \text { for each } x \text { in } \partial S_{R} .
$$

Lemma 2.5. Suppose that $u$ satisfies (2.1), $u_{R}$ satisfies (2.4), and $e_{R}=u-u_{R}$. Then there exists a constant $C$, independent of $R$, such that

$$
\left\|e_{R}\right\|_{H_{R}^{E}}^{2}<\frac{C}{R^{3}}\left(\oint_{\partial \Omega}\left|\frac{\partial u}{\partial n}\right|^{2} d s_{x}+\int_{\Omega_{R}}|f|^{2} d x\right),
$$

and

$$
\int_{\Omega_{R}}\left|e_{R}\right|^{2} d x \leqslant \frac{C}{R}\left(\oint_{\partial \Omega}\left|\frac{\partial u}{\partial n}\right|^{2} d s_{x}+\int_{\Omega_{R}}|f|^{2} d x\right)
$$


Proof. (a) It follows from (2.1), (2.4), and (2.7) that

$$
\frac{1}{R} \oint_{\partial S_{R}} e_{R}^{2} d s_{x}=-\oint_{\partial S_{R}} e_{R} \frac{\partial e_{R}}{\partial r} d s_{x}+\oint_{\partial S_{R}} e_{R} w_{R} d s_{x} \text {. }
$$

We apply the Schwarz inequality and Lemma 2.1(c) to the last term in (2.8) to deduce

$$
\begin{aligned}
\left|\oint_{\partial S_{R}} e_{R} w_{R} d s_{x}\right| & \leqslant\left(\oint_{\partial S_{R}} e_{R}^{2} d s_{x}\right)^{1 / 2}\left(\oint_{\partial S_{R}} w_{R}^{2} d s_{x}\right)^{1 / 2} \\
& \leqslant \frac{C}{R^{3}}\left(\oint_{\partial S_{R}} e_{R}^{2} d s_{x}\right)^{1 / 2}\left(\oint_{\partial \Omega}\left|\frac{\partial u}{\partial n}\right|^{2} d s_{x}+\int_{\Omega_{R}} f^{2} d x\right)^{1 / 2} \\
& \leqslant \frac{C_{\varepsilon}}{R^{3}}\left(\oint_{\partial \Omega}\left|\frac{\partial u}{\partial n}\right|^{2} d s_{x}+\int_{\Omega_{R}} f^{2} d x\right)+\frac{\varepsilon}{R} \oint_{\partial S_{R}} e_{R}^{2} d s_{x},
\end{aligned}
$$

where $\varepsilon>0$ is arbitrarily small and $C_{\varepsilon}$ depends on $\varepsilon$ but not on $R$. Applying (2.1), (2.4), and integration by parts, we see that

$$
\int_{\Omega_{R}}\left|\nabla e_{R}\right|^{2} d x-\oint_{\partial S_{R}} e_{R} \frac{\partial e_{R}}{\partial r} d s_{x}=-\int_{\Omega_{R}} e_{R} \Delta e_{R} d x-\oint_{\partial \Omega} e_{R} \frac{\partial e_{R}}{\partial n} d s_{x}=0
$$

Finally, we combine (2.8)-(2.10) to conclude that

$$
\begin{aligned}
\int_{\Omega_{R}}\left|\nabla e_{R}\right|^{2} d x+\frac{1}{R} \oint_{\partial S_{R}} e_{R}^{2} d s_{x} & =\int_{\Omega_{R}}\left|\nabla e_{R}\right|^{2} d x-\oint_{\partial S_{R}} e_{R} \frac{\partial e_{R}}{\partial r} d s_{x}+\oint_{\partial S_{R}} e_{R} w_{R} d s_{x} \\
& <\frac{C_{\varepsilon}}{R^{3}}\left(\oint_{\partial \Omega}\left|\frac{\partial u}{\partial n}\right|^{2} d s_{x}+\int_{\Omega_{R}} f^{2} d x\right)+\frac{\varepsilon}{R} \oint_{\partial S_{R}} e_{R}^{2} d s_{x} .
\end{aligned}
$$

We may now choose $\varepsilon=\frac{1}{2}$ in the last estimate to obtain (a).

(b) To begin with, we observe that

$$
\begin{aligned}
\int_{\Omega_{R}}\left|\nabla\left(r e_{R}\right)\right|^{2} d x & =\int_{\Omega_{R}}\left|r \nabla e_{R}+e_{R} \nabla r\right|^{2} d x \\
& =\int_{\Omega_{R}}\left(r^{2}\left|\nabla e_{R}\right|^{2}+e_{R}^{2}|\nabla r|^{2}+2 r e_{R} \nabla r \cdot \nabla e_{R}\right) d x
\end{aligned}
$$

Since $|\nabla r|=1$, we deduce

$$
\begin{aligned}
\int_{\Omega_{R}} e_{R}^{2} d x & =\int_{\Omega_{R}}\left|\nabla\left(r e_{R}\right)\right|^{2} d x-\int_{\Omega_{R}} r^{2}\left|\nabla e_{R}\right|^{2} d x-\int_{\Omega_{R}} 2 r e_{R} \nabla r \cdot \nabla e_{R} d x \\
& <\int_{\Omega_{R}}\left|\nabla\left(r e_{R}\right)\right|^{2} d x+\frac{1}{2} \int_{\Omega_{R}} e_{R}^{2} d x+C \int_{\Omega_{R}} r^{2}\left|\nabla e_{R}\right|^{2} d x
\end{aligned}
$$

Using (a), we obtain

$$
\int_{\Omega_{R}} r^{2}\left|\nabla e_{R}\right|^{2} d x<R^{2} \int_{\Omega_{R}}\left|\nabla e_{R}\right|^{2} d x<\frac{C}{R}\left(\oint_{\partial \Omega}\left|\frac{\partial u}{\partial n}\right|^{2} d s_{x}+\int_{\Omega_{R}} f^{2} d x\right) .
$$

Combining (2.11) and (2.12), we see that

$$
\int_{\Omega_{R}} e_{R}^{2} d x<\frac{C}{R}\left(\oint_{\partial \Omega}\left|\frac{\partial u}{\partial n}\right|^{2} d s_{x}+\int_{\Omega_{R}} f^{2} d x\right)+2 \int_{\Omega_{R}}\left|\nabla\left(r e_{R}\right)\right|^{2} d x
$$


We next estimate the last term in (2.13). We apply integration by parts to obtain

$$
\int_{\Omega_{R}}\left|\nabla\left(r e_{R}\right)\right|^{2} d x=\int_{\Omega_{R}} r e_{R}\left(-\Delta\left(r e_{R}\right)\right) d x+\oint_{\partial S_{R}} r e_{R} \frac{\partial}{\partial r}\left(r e_{R}\right) d s_{x} .
$$

To estimate the last term on the right, we observe that $r=R$ on $\partial S_{R}$ and we employ (2.8), (2.9), and (a) to conclude that

$$
\begin{aligned}
\left|\oint_{\partial S_{R}} r e_{R} \frac{\partial}{\partial r}\left(r e_{R}\right) d s_{x}\right| & =\left|\oint_{\partial S_{R}}\left(r e_{R}^{2}+r^{2} e_{R} \frac{\partial e_{R}}{\partial r}\right) d s_{x}\right|=\left|\oint_{\partial S_{R}} R^{2} e_{R} w_{R} d s_{x}\right| \\
& <R^{2}\left(\frac{C}{R^{3}}\left(\oint_{\partial \Omega}\left|\frac{\partial u}{\partial n}\right|^{2} d s_{x}+\oint_{\Omega_{R}} f^{2} d x\right)+\frac{1}{R} \oint_{\partial S_{R}} e_{R}^{2} d s_{x}\right) \\
& <\frac{C}{R}\left(\oint_{\partial \Omega}\left|\frac{\partial u}{\partial n}\right|^{2} d s_{x}+\int_{\Omega_{R}} f^{2} d x\right) .
\end{aligned}
$$

Finally, we estimate

$$
\int_{\Omega_{R}} r e_{R}\left(-\Delta\left(r e_{R}\right)\right) d x=\int_{\Omega_{R}} r e_{R}\left(e_{R}(-\Delta r)+r\left(-\Delta e_{R}\right)-2 \nabla r \cdot \nabla e_{R}\right) d x .
$$

Noting that $\Delta e_{R}=0$ and $\Delta r=2 / r$, we deduce

$$
\int_{\Omega_{R}} r e_{R}\left(-\Delta\left(r e_{R}\right)\right) d x=-2 \int_{\Omega_{R}}\left(e_{R}^{2}+r e_{R} \nabla r \cdot \nabla e_{R}\right) d x .
$$

Using (a), it follows readily that

$$
\begin{aligned}
\left|\int_{\Omega_{R}} 2 r e_{R} \nabla r \cdot \nabla e_{R} d x\right| & <\frac{1}{2} \int_{\Omega_{R}} e_{R}^{2} d x+C \int_{\Omega_{R}} r^{2}\left|\nabla e_{R}\right|^{2} d x \\
& <\frac{1}{2} \int_{\Omega_{R}} e_{R}^{2} d x+\frac{C}{R}\left(\oint_{\partial \Omega}\left|\frac{\partial u}{\partial n}\right|^{2} d s_{x}+\int_{\Omega_{R}} f^{2} d x\right) .
\end{aligned}
$$

Combining (2.14)-(2.17) with (2.13), we now conclude that

$$
\int_{\Omega_{R}} e_{R}^{2} d x<\frac{C}{R}\left(\oint_{\partial \Omega}\left|\frac{\partial u}{\partial n}\right|^{2} d s_{x}+\int_{\Omega_{R}} f^{2} d x\right) .
$$

This completes the proof of the lemma. Q.E.D.

The following result enables us to simplify the estimates in Lemma 2.5 .

Lemma 2.6. Suppose that $u$ satisfies (2.1). Then there exists a constant $C$, independent of $u$, such that

$$
\oint_{\partial \Omega}\left|\frac{\partial u}{\partial n}\right|^{2} d s_{x}<C \int_{\Omega} f^{2} d x
$$

Proof. Without loss of generality, we may assume that $\Omega \subset S_{1}$ and $\operatorname{supp}(f) \subset S_{1}$. Let $\chi$ denote a cutoff function satisfying the following conditions: $\chi \in C^{\infty}\left(\Omega^{c}\right)$, $\chi \equiv 1$ in a neighborhood of $\partial \Omega$ and $\chi \equiv 0$ in a neighborhood of $\partial S_{1}$. Set $\Omega_{1}=\Omega^{C}$ $\cap S_{1}$. Since $\chi u=0$ on $\partial \Omega$ and on $\partial S_{1}$, we may employ (2.1), the theory of elliptic differential equations and the triangle inequality, to deduce

$$
\begin{aligned}
\oint_{\partial \Omega}\left|\frac{\partial u}{\partial n}\right|^{2} d s_{x} & =\oint_{\partial \Omega}\left|\frac{\partial(\chi u)}{\partial n}\right|^{2} d s_{x}<C\|\chi u\|_{H^{2}\left(\Omega_{1}\right)}^{2} \\
& <C\|-\Delta(\chi u)\|_{L^{2}\left(\Omega_{1}\right)}^{2}<C\left(\|f\|_{L^{2}\left(\Omega^{c}\right)}^{2}+\|u\|_{H^{1}\left(\Omega_{1}\right)}^{2}\right) .
\end{aligned}
$$


Since $u=0$ on $\partial \Omega$, it follows that

$$
\int_{\Omega_{1}}|u|^{2} d x \leqslant C \int_{\Omega_{1}}|\nabla u|^{2} d x
$$

(see, e.g., [2]). We thus obtain

$$
\oint_{\partial \Omega}\left|\frac{\partial u}{\partial n}\right|^{2} d s_{x}<C\left(\int_{\Omega_{1}}|\nabla u|^{2} d x+\int_{\Omega^{c}} f^{2} d x\right) .
$$

Now suppose that $R_{0}$ is a fixed large positive number and $u_{R_{0}}$ satisfies (2.4) with $R$ replaced by $R_{0}$. Choosing $R_{0}$ sufficiently large, we may apply Lemma 2.5(a) to obtain

$$
\int_{\Omega_{R_{0}}}\left|\nabla\left(u-u_{R_{0}}\right)\right|^{2} d x \leqslant \frac{C}{R_{0}^{3}}\left(\oint_{\partial \Omega}\left|\frac{\partial u}{\partial n}\right|^{2} d s_{x}+\int_{\Omega^{c}} f^{2} d x\right),
$$

with $C$ independent of $R_{0}$. Again, employing elliptic regularity theory, we see that

$$
\left\|u_{R_{0}}\right\|_{H^{1}\left(\Omega_{R_{0}}\right)} \leqslant C_{R_{0}}\|f\|_{L^{2}\left(\Omega_{R_{0}}\right)}
$$

Combining (2.18)-(2.20), we conclude that

$$
\oint_{\partial \Omega}\left|\frac{\partial u}{\partial n}\right|^{2} d s_{x} \leqslant K_{R_{0}} \int_{\Omega c} f^{2} d x+\frac{C}{R_{0}^{3}} \oint_{\partial \Omega}\left|\frac{\partial u}{\partial n}\right|^{2} d s_{x},
$$

where $K_{R_{0}}$ depends on $R_{0}$ but $C$ is independent of $R_{0}$. We now choose $R_{0}^{3}=2 C$ to obtain the lemma. Q.E.D.

We may now combine Lemmas 2.5 and 2.6 to immediately deduce the main result of this section.

THeORem 2.1. Suppose that $R$ is sufficiently large and that $u$ and $u_{R}$ satisfy (2.1) and (2.4), respectively. Then there exists a constant $C$, independent of $R$ and $u$, such that

$$
\int_{\Omega_{R}}\left|\nabla\left(u-u_{R}\right)\right|^{2} d x+\frac{1}{R} \oint_{\partial S_{R}}\left(u-u_{R}\right)^{2} d s_{x}<\frac{C}{R^{3}} \int_{\Omega_{R}} f^{2} d x,
$$

and

$$
\int_{\Omega_{R}}\left(u-u_{R}\right)^{2} d x<\frac{C}{R} \int_{\Omega_{R}} f^{2} d x .
$$

Remark 2.1. There has recently been developed, [14], a hierarchy of approximate boundary conditions at infinity for calculating the solution of problems such as (2.1). The construction of these boundary conditions is based on the series expansion for $u$ given in Lemma 2.1(a). The first boundary condition in this hierarchy is given by $\partial u / \partial r+u / r=0$ on $\partial S_{R}$ and corresponds to the outer boundary condition in problem (2.4). The arguments of this paper appear to be applicable to higher order boundary conditions as well, although this will not be considered here.

We also observe that the following error estimate was proved in [14]:

$$
\left\|u-u_{R}\right\|_{L^{\infty}\left(\Omega_{R}\right)}<\frac{C}{R^{2}},
$$


where $C$ is independent of $R$. The proof of (2.22) is based on a version of the maximum principle dealing with impedence boundary conditions. Since we are analyzing the finite element method, which is based on a variational principle, the mean-square and energy estimates of Theorem 2.1 are more appropriate to the purposes of this paper.

3. The Finite Element Method. In this section we shall construct an approximate solution $u_{R}^{h}$ of (2.4) using the finite element method with element mesh size of order $O(h)$ on $\Omega_{R}$. We begin by considering a discretized version of the variational problem in Section 2, obtained by replacing $H_{R}^{E}$ by a finite-dimensional subspace. We shall then describe the finite element method and estimate the error $\left\|u-u_{R}^{h}\right\|_{H_{R}^{E}}$, where $u$ satisfies the original problem (2.1). We shall see that this error estimate is suboptimal, thus motivating the last two sections of the paper.

We begin with the following result.

LEMmA 3.1. Suppose that $R$ is sufficiently large, $u_{R}$ satisfies (2.4), $a_{R}($,$) and H_{R}^{E}$ are defined by (2.6), and $M$ is a finite-dimensional subspace of $H_{R}^{E}$. Then,

(a) there exists a unique solution $\hat{u}_{R} \in M$ of the equation

$$
a_{R}\left(\hat{u}_{R}, v\right)=(f, v) \quad \forall v \in M,
$$

and

(b) $\left\|u_{R}-\hat{u}_{R}\right\|_{H_{R}^{E}}=\inf _{\chi \in M}\left\|u_{R}-\chi\right\|_{H_{R}^{E}}$.

The proof of Lemma 3.1 follows easily from Lemma 2.4(a) using the fact that $\hat{u}_{R}$ is the projection of $u_{R}$ onto $M$ with respect to the inner product defined by $a_{R}($,$) ;$ see, e.g., [2] or [3]. We now describe how the finite element method may be employed to approximately solve problem (2.4). We begin by replacing $H_{R}^{E}$ by a one parameter family of finite-dimensional subspaces $S^{h}$, defined for each $h \in$ $(0, \infty)$. The subspaces $S^{h}$ are typically obtained by subdividing $\Omega_{R}$ into simple subsets (elements), denoted by $t^{h}$, with diameter of order $O(h) . S^{h}$ may then be defined as the subspace of $H_{R}^{E}$ consisting of all continuous functions $v^{h}$ such that the restriction of $v^{h}$ to each element $t^{h}$ belongs to some appropriate class of functions, denoted by $F$. For example, $F$ may consist of all polynomials of degree less than $K$ in a convenient coordinate system for a fixed integer $K$ not less than 2 .

Using Lemma 3.1, we may now define the finite element approximation $u_{R}^{h} \in S^{h}$ to the solution of problem (2.4) as the unique solution of

$$
a_{R}\left(u_{R}^{h}, v^{h}\right)=\left(f, v^{h}\right) \quad \forall v^{h} \in S^{h}
$$

We shall require certain approximation properties of our finite element spaces. Specifically, we assume that

$$
\left\{\begin{array}{l}
\inf _{x \in S^{h}} \int_{\Omega^{\prime}}|\nabla(v-\chi)|^{2} d x<C h^{2 s-2}|v|_{H^{s}\left(\Omega^{\prime}\right)}^{2} \\
\text { and } \\
\inf _{x \in S^{h}} \oint_{\partial S_{R}}|v-\chi|^{2} d s_{x}<C h^{2 s-1}|v|_{H^{s}\left(\Omega_{R}\right)}^{2}
\end{array}\right.
$$

for each $v \in H^{K}\left(\Omega_{R}\right)$ and each integer $s \in[2, K]$, where $K$ is an integer not less than 2 and $\Omega^{\prime}$ is an arbitrary subset of $\Omega_{R}$. The constant $C$ is independent of $\Omega^{\prime}, h$, 
and $v$. It is important to note that only derivatives of order $s$ appear on the right-hand side of these estimates. The estimates in (3.3) hold for typical finite element spaces; see, e.g., [1]-[3].

There is another condition, typically satisfied by finite element subspaces, that will be useful throughout this paper. Let $\Omega^{\prime}$ denote an arbitrary subset of $\Omega_{R}$. Set $S_{\Omega^{\prime}}^{h^{\perp}}=\left\{\chi \in S^{h}: \chi(x)=0\right.$ for $\left.x \in \Omega^{\prime}\right\}$ and denote the orthogonal complement of $S_{\Omega^{\prime}}^{h^{\perp}}$ by $S_{\Omega^{\prime}}^{h}=S^{h}-S_{\Omega^{\prime}}^{h^{\perp}}$. We assume that there exist constants, $C_{1}$ and $C_{2}$, independent of $h \in(0,1]$ and $\Omega^{\prime}$, such that

$$
C_{1} h^{-3} \operatorname{meas}\left(\Omega^{\prime}\right) \leqslant \operatorname{dim}\left(S_{\Omega^{\prime}}^{h}\right)<C_{2} h^{-3} \operatorname{meas}\left(\Omega^{\prime}\right) .
$$

Here meas $\left(\Omega^{\prime}\right)$ denotes the measure of $\Omega^{\prime}$ and $\operatorname{dim}\left(S_{\Omega^{\prime}}^{h}\right)$ denotes the dimension of the finite-dimensional subspace $S_{\Omega^{\prime}}^{h}$.

Remark 3.1. We observe that since $S^{h} \subset H_{R}^{E}$, functions in $S^{h}$ must vanish on $\partial \Omega$. This will cause problems, in general, when $\partial \Omega$ does not have a simple shape. (The boundary condition on $\partial S_{R}$ is natural. Hence, no boundary condition needs to be imposed on $\partial S_{R}$.) Methods have been developed for overcoming the difficulties associated with essential boundary conditions; see [1] or [2]. For example, $\partial \Omega$ may be approximated by surfaces with simple shapes or the finite element method may be generalized. These techniques for treating essential boundary conditions on $\partial \Omega$ will not affect the arguments of this paper. For the sake of simplicity, we shall consider the usual finite element formulation described above. See [1]-[3] for more detailed descriptions of the finite element method.

We next estimate the error $u-u_{R}^{h}$ in the energy norm.

THEOREM 3.1. Suppose that the hypotheses of Lemma 3.1 hold with $M$ replaced by $S_{R}^{h}$ for $h \in(0,1]$, condition (3.3) holds with $K>2$, and $u$ satisfies (2.1). Then there exists a unique solution $u_{R}^{h} \in S^{h}$ of (3.2) and a constant $C$, independent of $u, h$, and $R$, such that

$$
\left\|u-u_{R}^{h}\right\|_{H_{R}^{E}} \leqslant C\left(R^{-3 / 2}\|f\|_{L^{2}\left(\Omega^{C}\right)}+h^{K-1}|u|_{H^{\kappa}\left(\Omega_{R}\right)}\right) .
$$

Proof. We may apply Lemma 3.1 and Theorem 2.1(a) to see that there exists a unique solution $u_{R}^{h} \in S^{h}$ of (3.2) such that

$$
\begin{aligned}
\left\|u-u_{R}^{h}\right\|_{H_{R}^{E}} & \leqslant\left\|u-u_{R}\right\|_{H_{R}^{E}}+\left\|u_{R}-u_{R}^{h}\right\|_{H_{R}^{E}} \\
& \leqslant C R^{-3 / 2}\|f\|_{L^{2}\left(\Omega^{c}\right)}+\inf _{\chi \in S^{h}}\left\|u_{R}-\chi\right\|_{H_{R}^{E}} \\
& \leqslant 2 C R^{-3 / 2}\|f\|_{L^{2}\left(\Omega^{c}\right)}+\inf _{\chi \in S^{h}}\|u-\chi\|_{H_{R}^{E}}
\end{aligned}
$$

Combining this estimate with (3.3), we have proved the theorem. Q.E.D.

We may obtain an $L^{2}$ estimate for $u-u_{R}^{h}$ in an analogous fashion using Theorem 2.1 and a duality argument. We postpone the details of this argument until Section 5 since both the energy and $L^{2}$ estimates are suboptimal when a uniform mesh size is employed, as in this section. We shall demonstrate the suboptimality of estimate (3.5) as follows. First observe from (3.5) that in order to obtain the estimate

$$
\left\|u-u_{R}^{h}\right\|_{H_{R}^{E}}<C h^{K-1}
$$


it suffices to make $R$ sufficiently large that

$$
R^{-3 / 2}=h^{K-1} \text {. }
$$

Estimate (3.6) does not tell the whole story since the number of equations to be solved to obtain $u_{R}^{h}$ is expressed in terms of $\operatorname{dim}\left(S^{h}\right)$. We define a new parameter $H$ by

$$
H=\left(\operatorname{dim}\left(S^{h}\right)\right)^{-1 / 3} \text {. }
$$

$H$ is often taken as the relevent parameter to measure convergence when using the finite element method; see [1]. In view of assumption (3.4), we see that

$$
C_{1} h^{-3} R^{3}<\operatorname{dim}\left(S^{h}\right)<C_{2} h^{-3} R^{3} .
$$

It follows from (3.7) that $R=h^{-2(K-1) / 3}$. Hence we see, using (3.8) and (3.9), that

$$
C_{2}^{-1 / 3} h^{(2 K+1) / 3} \leqslant H \leqslant C_{1}^{-1 / 3} h^{(2 K+1) / 3} \text {. }
$$

We may thus employ Theorem 3.1 to deduce

Corollary 3.1. Suppose that (3.4) and the hypotheses of Theorem 3.1 hold. Then there exists a constant $C$, independent of $h$, such that, for $R=h^{-2(K-1) / 3}$, we have

$$
\left\|u-u_{R}^{h}\right\|_{H_{R}^{E}} \leqslant C H^{3(K-1) /(2 K+1)} \text {. }
$$

Remark 3.2. Suppose that we replace (2.1) by the following problem

(3.11) $(-\Delta+\lambda) u=f$ in $\Omega^{C}, \quad u=0$ on $\partial \Omega, \quad u \in L^{2}\left(\Omega^{C}\right), \lambda>0, f \in C_{0}^{\infty}\left(\Omega^{C}\right)$.

It can then be shown that $D^{\alpha} u(x)=O\left(e^{-\sigma|x|}\right)$ for some $\sigma>0$ and all $|x|$ sufficiently large. From this, it may be seen, using the arguments and assumptions of this section, that

$$
\left\|u-u_{R}^{h}\right\|_{H_{R}^{E}} \leqslant C_{\varepsilon} H^{K-1 \varepsilon} \text { for arbitrary } \varepsilon>0,
$$

with $u_{R}^{h}$ defined as before. An analogous estimate was obtained by Babuška in [1] and [4] for a problem similar to (3.11).

We shall refer to the following estimates as optimal for problems in unbounded domains:

$$
\left\|u-u_{R}^{h}\right\|_{H_{R}^{E}} \leqslant C H^{K-1},
$$

and

$$
\left\|u-u_{R}^{h}\right\|_{L^{2}(B)}<C H^{K},
$$

where $B$ is a fixed bounded subset of $\Omega^{C}$. (For problems on bounded domains, optimality is generally defined by estimates such as (3.12) and (3.13).) We see from Corollary 3.1 that the energy estimate is considerably weaker than optimal for problem (2.1). It may also be readily seen that $L^{2}$ estimates are suboptimal when uniform mesh sizes are employed. We shall show in Sections 4 and 5 that the optimal estimates (3.12) and (3.13), respectively, hold when the mesh is graded systematically in such a way that the mesh sizes of elements "near infinity" become large.

4. Energy Estimates. It is our purpose, in this section, to prove an optimal energy estimate of the form (3.12). We shall accomplish this by replacing the family of finite element spaces $S^{h}$, described in Section 3, by a new family of spaces $\tilde{S}^{h}$ 
obtained by a mesh grading process to be described below. We begin by constructing the family of spaces $\tilde{S}^{h}$ in such a way that the following approximation estimate holds:

$$
\inf _{x \in \tilde{S}^{h}}\|u-\chi\|_{H_{R}^{E}}<C H^{K-1}
$$

where $H$ is defined by (3.8) and $u$ satisfies (2.1). We shall then combine this with the results of Sections 2 and 3 to obtain our optimal error estimate. At the end of the section we shall illustrate the mesh grading process with respect to a specific example.

To begin with, suppose that we have a family of finite element spaces $S^{\boldsymbol{h}} \subset \boldsymbol{H}_{R}^{E}$ satisfying conditions (3.3) and (3.4) for each $h \in(0, \infty)$. Hence, in particular, we assume that $\Omega_{R}$ is partitioned into a union of elements $t^{h}$ with diameter of order $O(h)$. Functions in $S^{h}$ are continuous and are such that their restriction to each $t^{h}$ belongs to a simple class of functions, denoted by $F$, as described in Section 3 . We now define a new subspace $\tilde{S}^{h} \subset H_{R}^{E}$ by systematically increasing the mesh sizes of elements as their distance from the origin increases in such a way that estimate (4.1) will hold.

To be precise, we define

$$
S_{j}=\left\{x: 2^{j-1}<|x|<2^{j}\right\}, \quad j=1,2, \ldots, J_{R},
$$

with $J_{R}$ to be specified below. Without loss of generality, we may assume that $\Omega$ and the support of $f$ are contained in the unit sphere. Set $\Omega_{j}^{R}=S_{j} \cap \Omega_{R}, j=$ $1,2, \ldots, J_{R}$, and $\Omega_{0}^{R}=\bar{\Omega}_{R}-\cup_{j=1}^{J_{R}} \Omega_{j}^{R}$. We may obtain a new partition of $\Omega_{R}$ by assuming that elements $t^{h_{j}}$ in each annular region $\Omega_{j}^{R}$ have diameter of order $O\left(h_{j}\right)$, with $h_{j}$ to be determined shortly, $j=0,1, \ldots, J_{R}$. We now define $\tilde{S}^{h}$ to consist of those continuous functions $\chi \in H_{R}^{E}$ such that $\chi$ restricted to each $t^{h_{3}}$ belongs to $F$.

In order to satisfy (4.1), we determine the parameters $h_{j}$ as follows. Using (3.3), we may choose a function $\chi_{j} \in S^{h_{j}}$ such that

$$
\left\{\begin{array}{l}
\int_{\Omega_{j}} R\left|\nabla\left(u-\chi_{j}\right)\right|^{2} d x \leqslant C h_{j}^{2 K-2}|u|_{H^{\kappa}\left(\Omega_{j}^{R}\right)}^{2}, \quad j=0,1, \ldots, J_{R}, \\
\text { and } \\
\frac{1}{R} \oint_{\partial S_{R}}\left|u-\chi_{J_{R}}\right|^{2} d s_{x} \leqslant C \frac{h_{J_{R}}^{2 K-1}}{R}|u|_{H^{K}\left(\Omega_{J_{R}}^{R}\right)}^{2}
\end{array}\right.
$$

where $C$ is independent of $h_{j}, u$, and $R$. We shall next apply Lemma 2.1. For the sake of simplicity, we assume in the remainder of this paper that Lemma 2.1 holds for $r=|x| \geqslant 1$. We employ Lemma 2.1(b) to see that

$$
|u|_{H^{K}\left(\Omega_{j}^{R}\right)}^{2} \leqslant C \int_{\Omega_{j}^{R}} r^{-2(K+1)} d x \leqslant C 2^{-(2 K-1) j} .
$$

In view of (4.2) and (4.3), we wish to choose $h_{j}$ such that

$$
h_{j}^{2 K-2} 2^{-(2 K-1) j}<h^{2 K-2} \text {. }
$$

Hence, we set

$$
h_{0}=h \quad \text { and } \quad h_{j}=2^{K^{\prime} h} h \quad \text { with } 1<K^{\prime}<\frac{K-1 / 2}{K-1}, j=1,2, \ldots, J_{R} .
$$


We next choose $J_{R}^{\prime}$ so that $2^{V_{R}^{\prime}}=R$. For the reasons given in Section 3, we define $R$ by (3.7). We thus have

$$
R=h^{-2(K-1) / 3},
$$

so that $J_{R}^{\prime}=\log _{2}(1 / h)^{2(K-1) / 3}$. Since $J_{R}^{\prime}$ is not necessarily an integer, set

$$
J_{R}=\left[J_{R}^{\prime}\right]+1=\left[\log _{2}\left(\frac{1}{h}\right)^{2(K-1) / 3}\right]+1,
$$

where $\left[J_{R}^{\prime}\right]$ denotes the greatest integer less than $J_{R}^{\prime}$. Set $\sigma=J_{R}-J_{R}^{\prime}$ and note that

$$
2^{J_{R}}=2^{\sigma} R, \quad 0<\sigma<1 .
$$

Combining (4.5), (4.6), and (4.8), we readily deduce

$$
h_{J_{R}}<R
$$

for $h$ sufficiently small.

We now let $\chi$ denote the function in $\tilde{S}^{h}$ such that $\chi$, restricted to $\Omega_{j}^{R}$, is equal to $\chi_{j}$, where $\chi_{j}$ satisfies $(4.2), j=0,1, \ldots, J_{R}$. Hence, we may combine (4.2), (4.3), (4.5), and (4.9) to obtain

$$
\begin{aligned}
\|u-\chi\|_{H_{R}^{E}} & \leqslant \sum_{j=0}^{J_{R}} C h_{j}^{K-1} 2^{-(K-1 / 2) j} \\
& \leqslant C \sum_{j=0}^{J_{R}} h^{K-1} 2^{K^{\prime}(K-1) j} 2^{-(K-1 / 2) j}<C h^{K-1},
\end{aligned}
$$

where $h$ is sufficiently small and the constant $C$ is independent of $h$. Furthermore, we may apply (3.4) and (4.5) to deduce

$$
\begin{aligned}
\operatorname{dim}\left(\tilde{S}^{h}\right) & <\sum_{j=0}^{J_{R}} \operatorname{dim}\left(\tilde{S}_{\Omega_{j}^{R}}^{h}\right)<C \sum_{j=0}^{J_{R}} h_{j}^{-3} \operatorname{meas}\left(\Omega_{j}^{R}\right) \\
& <C \sum_{j=0}^{J_{R}} h_{j}^{-3}\left(2^{j}\right)^{3}<C\left(h^{-3}+\sum_{j=1}^{J_{R}} h^{-3}\left(2^{-K^{\prime} j}\right)^{3}\left(2^{j}\right)^{3}\right) \\
& <C h^{-3} \sum_{j=0}^{J_{R}}\left(2^{-3\left(K^{\prime}-1\right)}\right)^{j}<C_{2} h^{-3},
\end{aligned}
$$

where $h$ is sufficiently small and the constant $C_{2}$ is independent of $h$. In view of (3.4) and (4.5), we also readily obtain

$$
\operatorname{dim}\left(\tilde{S}^{h}\right) \geqslant C_{1} h^{-3},
$$

with $h$ sufficiently small and $C_{1}$ independent of $h$. Combining (3.8), (4.11), and (4.12), we deduce

$$
K_{1} h<H \leqslant K_{2} h,
$$

for $h$ sufficiently small and suitable constants $K_{1}$ and $K_{2}$ independent of $h$. Finally, we combine (4.10) and (4.13) to conclude that (4.1) holds.

We next apply Lemma 3.1 to obtain a unique function $\tilde{u}_{R}^{h} \in \tilde{S}^{h}$ satisfying

$$
a_{R}\left(\tilde{u}_{R}^{h}, v^{h}\right)=\left(f, v^{h}\right) \quad \forall v^{h} \in \tilde{S}^{h} .
$$


Applying the triangle inequality, Theorem 2.1(a), and Lemma 3.1, we see that

$$
\begin{aligned}
\left\|u-\tilde{u}_{R}^{h}\right\|_{H_{R}^{E}} & \leqslant\left\|u-u_{R}\right\|_{H_{R}^{E}}+\left\|u_{R}-\tilde{u}_{R}^{h}\right\|_{H_{R}^{E}} \\
& \leqslant C R^{-3 / 2}+\inf _{x \in \tilde{S}^{h}}\left\|u_{R}-\chi\right\|_{H_{R}^{E}}<C R^{-3 / 2}+\inf _{\chi \in \tilde{S}^{h}}\|u-\chi\|_{H_{R}^{E}}
\end{aligned}
$$

Finally, we combine (4.1), (4.6), and (4.13) with (4.15) to obtain the main result of this section.

THEOREM 4.1. Suppose that $\tilde{S}^{h}$ is the subspace of $H_{R}^{E}$ defined above with $R=$ $h^{-2(K-1) / 3}, u$ satisfies $(2.1)$, and $\tilde{u}_{R}^{h}$ satisfies (4.14). Then there exists an $h_{0} \in(0,1]$ such that, for $h \in\left(0, h_{0}\right]$, we have

$$
\left\|u-\tilde{u}_{R}^{h}\right\|_{H_{R}^{E}} \leqslant C H^{K-1},
$$

where $C$ is independent of $h$ and $H$ is defined by (3.8).

Remark 4.1. Observe that we assumed $R$ and $h$ to be related by (4.6) in order to obtain an optimal error estimate for $u-\tilde{u}_{R}^{h}$. However, the mesh grading process was defined independently of $R$. The main feature of this process is embodied in the following estimate:

$$
\operatorname{diam}(\tau)<h d^{(K-1 / 2) /(K-1)} \text { on each annulus } \Omega_{j}^{R},
$$

where $d$ is the distance of the element $\tau$ from the origin. Note that if the mesh size is roughly doubled on successive annuli, so that $h_{j}=2^{j} h$, then $\operatorname{dim}\left(\tilde{S}^{h}\right)=$ $O\left(h^{-3}|\log h|\right)$ instead of $O\left(h^{-3}\right)$.

Remark 4.2. The results of this paper may be extended in various directions. For example, these methods may be applied to problems in scattering theory by replacing the Laplace operator by the Helmholtz operator, given by $-\Delta-K^{2}$. In this case, the condition at infinity is replaced by a suitable radiation condition. There are, however, certain technical difficulties associated with the indefiniteness of the relevent bilinear form. We may also treat variable coefficient perturbations of the Laplace and Helmholtz operators by the present methods, provided the perturbations have bounded support. In a subsequent publication, we shall deal with these and other extensions of the present results (such as $L^{\infty}$ error estimates). Finally, we mention that numerical computations demonstrating the theoretical results of this paper will appear elsewhere.

Example 4.1. In order to clarify the finite element method described above, we consider an axially symmetric problem in the exterior of the sphere $\Omega=\{x$ : $\left.|x|<\frac{1}{2}\right\}$. Using spherical polar coordinates and the axial symmetry, we may replace $\Omega_{R}=\left\{x: \frac{1}{2} \leqslant|x| \leqslant R\right\}$ by the following two-dimensional domain:

$$
\hat{\Omega}_{R}=\left\{(r, \theta): \frac{1}{2}<r<R, 0<\theta<\pi\right\} .
$$

The solution $u_{R}$ of problem (2.4) now satisfies the following boundary conditions on $\partial \hat{\Omega}_{R}$ :

$$
u_{R}=0 \quad \text { on } r=\frac{1}{2}, \quad \frac{\partial u_{R}}{\partial r}+\frac{1}{R} u_{R}=0 \quad \text { on } r=R, \quad \text { and } \frac{\partial u_{R}}{\partial \theta}=0 \quad \text { at } \theta=0, \pi \text {. }
$$

The last condition is due to the axial symmetry. The differential operator $-\Delta$ as well as the bilinear form $a_{R}($,$) are readily expressed in polar coordinates.$ 
We now construct a finite element space $S^{h}$ consisting of piecewise linear functions defined on $\hat{\Omega}_{R}$. We first partition $\hat{\Omega}_{R}$ into triangles of maximum diameter $h$, as shown in Figure 4.1, taking a uniform mesh spacing in the $r$ and $\theta$ directions. Next, let $S^{h}$ consist of all functions $\chi(r, \theta)$, defined on $\hat{\Omega}_{R}$, satisfying the following conditions:

(i) $\chi$ is continuous on $\hat{\Omega}_{R}$,

(ii) $\chi$ is linear on each triangle, and

(iii) $\chi=0$ on $r=\frac{1}{2}$.

The space $S^{h}$ may be constructed using a nodal basis of Lagrange type in the usual way; see, e.g., [2] or [3]. It may be readily seen that $S^{h}$ satisfies (3.3) (with $K=2$ ) and (3.4). In Figure 4.1, we set $h_{r}=h_{0}=2^{-3}$ and $h_{\theta}=\pi / 10$.

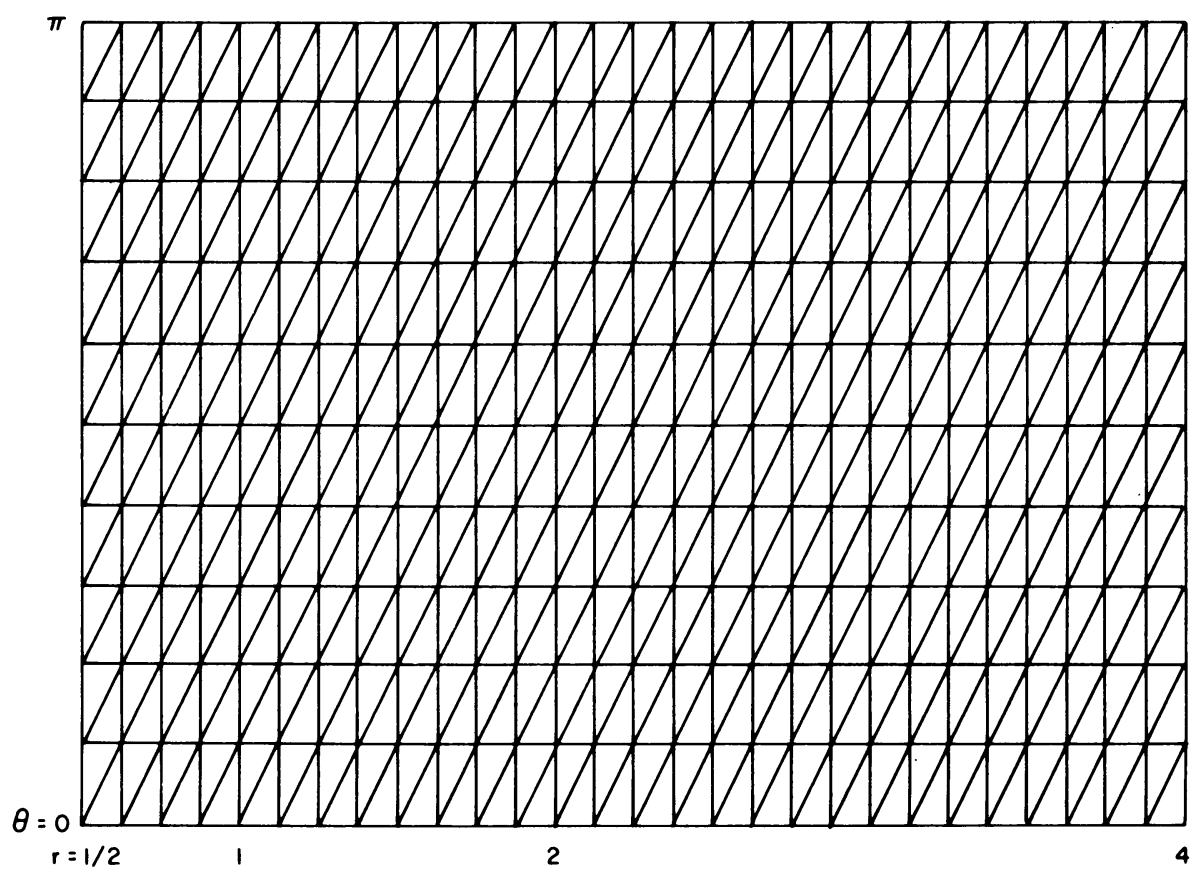

FIGURE 4.1 (Uniform Mesh)

We next define a new finite element space $\tilde{S}^{h}$ in accordance with the mesh grading procedure described above; see Figure 4.2. For simplicity, suppose that $\operatorname{supp}(f) \subset\{x:|x|<1\}$. We set $\Omega_{0}^{R}=\left\{x: \frac{1}{2}<|x|<1\right\}, \Omega_{j}^{R}=\left\{x: 2^{j-1}<|x|<\right.$ $\left.2^{j}\right\}, j=1,2, \ldots, J_{R}, h_{\theta}=\pi / 10$, and $h_{0}=2^{-3}$. We increase the mesh size in the $r$ direction as described above. Hence, we set $h_{j r}=2^{3 j / 2} h_{0}, j=0,1, \ldots, J_{R}$. Using (4.6) and (4.7), respectively, we set $R=2^{2}$ and $J_{R}=2$. Functions in $\tilde{S}^{h}$ are now defined with respect to this partition in the same manner as functions in $S^{h}$. 


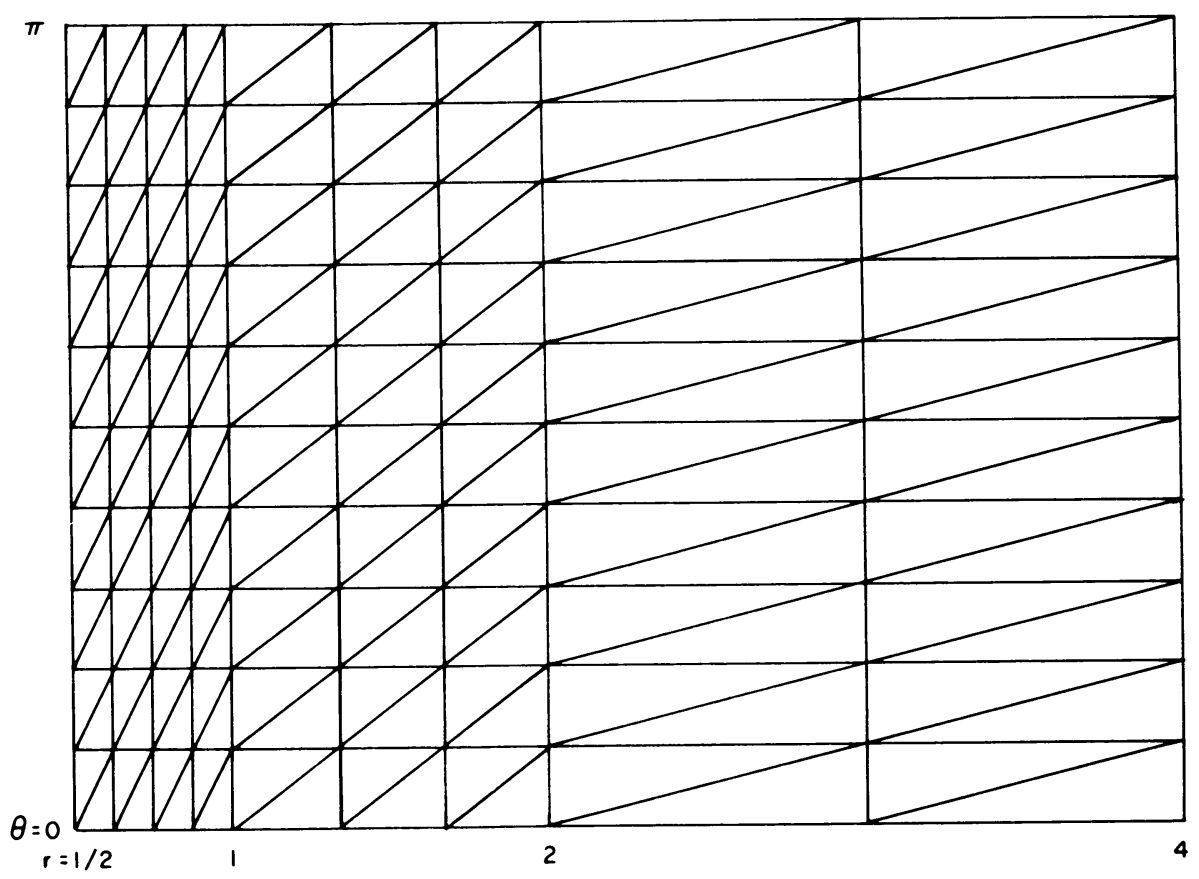

FIGURE 4.2 (Nonuniform Mesh)

5. $L^{2}$ Estimates. In this section, we shall establish the following optimal meansquare error estimate:

$$
\left\|u-\tilde{u}_{R}^{h}\right\|_{L^{2}(B)}<C H^{K},
$$

where $B$ is a bounded subset of $\Omega^{C}$ and $\tilde{u}_{R}^{h}$ is defined by (4.14). Note that $u, H$, and $K$ are defined as before. We first show that, by combining the results of Sections 2 and 3 with a duality argument, we may reduce our mean-square estimate to an approximation estimate. We shall then show that the mesh grading process described in Section 4 yields our optimal mean-square error estimate.

We may assume without loss of generality that $\Omega$ and the support of $f$ are contained in the unit sphere and Lemma 2.1 holds for $r>1$. In order to obtain estimate (5.1) we shall require the following lemma.

LEMmA 5.1. Suppose that $B$ is a bounded subset of $\Omega^{C}, R$ is sufficiently large, $u_{R}$ satisfies (2.4), $a_{R}\left(\right.$, ) and $H_{R}^{E}$ are defined by (2.6), $M$ is a finite-dimensional subspace of $H_{R}^{E}$, and $\hat{u}_{R} \in M$ satisfies the equation

$$
a_{R}\left(\hat{u}_{R}, v\right)=(f, v) \quad \forall v \in M .
$$

Then

$$
\left\|u_{R}-\hat{u}_{R}\right\|_{L^{2}(B)}<\inf _{\chi \in M}\left\|u_{R}-\chi\right\|_{H_{R}^{E}}\left(\sup _{\phi \in C_{0}^{\infty}(B)} \frac{1}{\|\phi\|_{L^{2}(B)}} \inf _{\psi \in M}\left\|\Phi_{R}-\psi\right\|_{H_{R}^{E}}\right),
$$

where $\Phi_{R}$ and $\phi$ are related by

(5.3) $-\Delta \Phi_{R}=\phi$ in $\Omega_{R}, \quad \Phi_{R}=0$ on $\partial \Omega$ and $\frac{\partial \Phi_{R}}{\partial r}+\frac{1}{R} \Phi_{R}=0$ on $\partial S_{R}$. 
Proof. It follows from the definition of the $L^{2}$ norm that

$$
\left\|u_{R}-\hat{u}_{R}\right\|_{L^{2}(B)}=\sup _{\phi \in C_{0}^{\infty}(B)} \frac{\left|\left(u_{R}-\hat{u}_{R}, \phi\right)\right|}{\|\phi\|_{L^{2}(B)}} .
$$

Combining (2.4), (2.6), (5.2), (5.3), and integration by parts, we see that

$$
\left(u_{R}-\hat{u}_{R}, \phi\right)=a_{R}\left(u_{R}-\hat{u}_{R}, \Phi_{R}\right)=a_{R}\left(u_{R}-\hat{u}_{R}, \Phi_{R}-\psi\right) \quad \forall \psi \in M .
$$

Hence, we may apply the Schwarz inequality to obtain

$$
\left|\left(u_{R}-\hat{u}_{R}, \phi\right)\right| \leqslant\left\|u_{R}-\hat{u}_{R}\right\|_{H_{R}^{E}} \inf _{\psi \in M}\left\|\Phi_{R}-\psi\right\|_{H_{R}^{E}}
$$

Finally, we may employ Lemma 3.1(b), (5.4), and (5.5) to obtain the lemma. Q.E.D.

We next apply Lemma 2.5(b) and Lemma 5.1 to the family of finite element spaces $\tilde{S}^{h}$ defined in Section 4 . Hence, we assume that $h_{j} \leqslant 2^{K^{\prime}} h, j=0,1, \ldots, J_{R}$, with $K^{\prime}$ defined in (4.5). Now we make the additional assumption that each $h_{j} \leqslant R$. Set

$$
R^{-1 / 2}=h^{K}
$$

The integer $J_{R}$ may be calculated as in Section 4 using (5.6). We thus obtain $J_{R}=\left[\log _{2}(1 / h)^{2 K}\right]+1$. It also follows, as in Section 4 , that $\operatorname{dim}\left(\tilde{S}^{h}\right)=O\left(h^{-3}\right)$ for $h$ sufficiently small.

We recall that $\tilde{u}_{R}^{h}$ is defined as the unique solution of Eq. (4.14). Using the triangle inequality, we obtain

$$
\left\|u-\tilde{u}_{R}^{h}\right\|_{L^{2}(B)} \leqslant\left\|u-u_{R}\right\|_{L^{2}(B)}+\left\|u_{R}-\tilde{u}_{R}^{h}\right\|_{L^{2}(B)} .
$$

We thus see, from Lemma 2.5(b) and (5.6), that

$$
\left\|u-\tilde{u}_{R}^{h}\right\|_{L^{2}(B)} \leqslant C R^{-1 / 2}+\left\|u_{R}-\tilde{u}_{R}^{h}\right\|_{L^{2}(B)}<C h^{K}+\left\|u_{R}-\tilde{u}_{R}^{h}\right\|_{L^{2}(B)} .
$$

We next apply Lemma 5.1, with $M$ replaced by $\tilde{S}^{h}$, to the last term in (5.7). In view of this, our goal is to prove the following two estimates:

$$
\inf _{x \in \tilde{S}^{h}}\left\|u_{R}-x\right\|_{H_{R}^{E}}<C h^{K-1}
$$

and

$$
\inf _{\psi \in \tilde{S}^{h}}\left\|\Phi_{R}-\psi\right\|_{H_{R}^{E}}<C h\|\phi\|_{L^{2}(B)}
$$

Employing Theorem 2.1(a) and the triangle inequality, we readily see that it suffices to prove

and

$$
\inf _{\chi \in \tilde{S}^{h}}\|u-\chi\|_{H_{R}^{E}}<C h^{K-1}
$$

where $\Phi$ satisfies

$$
\text { (5.10) }-\Delta \Phi=\phi \quad \text { in } \Omega^{C}, \quad \Phi=0 \text { on } \partial \Omega \text { and } \frac{\partial \Phi}{\partial r}+\frac{1}{r} \Phi=o\left(\frac{1}{r}\right) \quad \text { as } r \rightarrow \infty \text {. }
$$

The proof of estimate (5.8) follows as in Section 4. To prove (5.9), we may apply Lemma 2.1(b), Lemma 2.6, and the arguments of Section 4 (with $K$ replaced by 2). 
We thus obtain the following sufficient condition for (5.9) to hold:

$$
h_{j} \leqslant 2^{K^{\prime \prime}} h, \quad j=1,2, \ldots, J_{R}, \text { with } K^{\prime}<K^{\prime \prime}<\frac{3}{2} .
$$

Since $2^{K^{\prime} j}<2^{K^{\prime \prime} j}$, it is readily seen that (5.11) holds. Combining (5.7) with Lemma 5.1, (5.8), and (5.9), we obtain our main result as in Section 4.

THEOREM 5.1. Suppose that $B$ is a bounded subset of $\Omega^{C}, \tilde{S}^{h}$ is the subspace of $H_{R}^{E}$ defined in Section 4 with $R=h^{-2 K}$, u satisfies (2.1), and $\tilde{u}_{R}^{h}$ satisfies (4.14). Then there exists an $h_{0} \in(0,1]$ such that, for $h \in\left(0, h_{0}\right]$, we have

$$
\left\|u-u_{R}^{h}\right\|_{L^{2}(B)} \leqslant C H^{K},
$$

where $C$ is independent of $h$ and $H$ is defined by (3.8).

Acknowledgment. The author wishes to express his thanks to Professor A. Schatz for useful discussions concerning mesh refinements.

Applied Mathematics Department

Brookhaven National Laboratory

Upton, New York 11973

1. I. BABušKa \& A. K. AzIz, "Survey lectures on the mathematical foundations of the finite element method," The Mathematical Foundations of the Finite Element Method with Applications to Partial Differential Equations (A. K. Aziz, Ed.), Academic Press, New York, 1972.

2. P. G. CinRlEt, The Finite Element Method for Elliptic Problems, North-Holland, Amsterdam, 1978.

3. G. Strang \& G. Fix, An Analysis of the Finite Element Method, Prentice-Hall, Englewood Cliffs, N. J., 1973. $1-11$.

4. I. BabušKa, "The finite element method for infinite domains. I," Math. Comp., v. 26, 1972, pp.

5. J. Giroire \& J. C. Nedelec, "Numerical solutions of an exterior Neumann problem using a double layer potential," Math. Comp., v. 32, 1978, pp. 973-990.

6. S. MArin, A Finite Element Method for Problems Involving the Helmholtz Equation in Two Dimensional Exterior Regions, Thesis, Carnegie-Mellon University, Pittsburgh, Pa., 1978.

7. A. H. SChATZ \& L. B. WAHLBIN, "Maximum norm estimates in the finite element method on plane polygonal domains. Part 2, Refinements," Math. Comp., v. 33, 1979, pp. 465-492.

8. I. BABUŠKর, "Finite element method for domains with corners," Computing, v. 6, 1970, pp. 264-273.

9. S. C. Eisenstat \& M. H. Schultz, "Computational aspects of the finite element method," The Mathematical Foundations of the Finite Element Method (A. K. Aziz, Ed.), Academic Press, New York, 1972, pp. 505-524.

10. R. W. THATCHER, "The use of infinite grid refinements at singularities in the solution of Laplace's equation," Numer. Math., v. 25, 1976, pp. 163-178.

11. O. D. Kellogg, Foundations of Potential Theory, Ungar, New York, 1929.

12. G. C. Hsaio \& R. C. MACCAMY, "Solution of boundary value problems by integral equations of the first kind," SIAM Rev., v. 15, 1973, pp. 687-705.

13. M. SCHECHTER, "General boundary value problems for elliptic partial differential equations," Comm. Pure Appl. Math., v. 12, 1959, pp. 457-486.

14. A. BAyuss, M. GUNZBerger \& E. TURKRL, Boundary Conditions for the Numerical Solution of Elliptic Equations in Exterior Regions, ICASE Report 80-1, 1979. 